\title{
Tapes philippinarum seed exposure to metals in polluted areas of the Venice lagoon
}

\author{
A. Sfriso*, E. Argese, C. Bettiol, C. Facca \\ Department of Environmental Sciences, University of Venice, Calle Larga, Santa Marta, 2137, 30123 Venice, Italy
}

\section{A R T I C L E I N F O}

\section{Article history:}

Received 20 February 2008

Accepted 26 May 2008

Available online 17 June 2008

\section{Keywords:}

Tapes philippinarum

clam growth

metals

bioaccumulation

lagoon of Venice

\section{Introduction}

The introduction of the edible species Tapes philippinarum in the lagoons of the northern Adriatic Sea has played a key role in the fishery resources of the coastal areas. Tapes philippinarum, characterized by a rapid growth and spread, soon colonized the lagoons and estuaries of the Venice region, growing mainly in the inner areas affected by trophic inflows. Those areas display high nutrient, organic matter, phytoplankton and microphytobenthos concentrations (Facca et al., 2002a,b; Sfriso et al., 2003, 2005d) which favour clam growth but, unfortunately, also exhibit high concentrations of pollutants such as some metals (Donazzolo et al., 1984; Marcomini et al., 1997; Bellucci et al., 2002; Bernardello et al., 2006).

Recent studies on the Venice lagoon showed that some toxic elements, such as $\mathrm{Hg}$ and As, are widespread and exhibit high concentrations in surface sediments (Critto and Marcomini, 2001; Bellucci et al., 2002 and references therein; Bernardello et al., 2006). Tapes philippinarum spread in wide areas, which show a high contamination risk and have been precluded to fishing. Those areas are located near the mainland in proximity of Porto Marghera industries and urban waste outflows. Sediments and particulate

\footnotetext{
* Corresponding author.

E-mail address: sfrisoad@unive.it (A. Sfriso).
}

matter are also potential sources of contaminants. In particular, studies on the settled particulate matter (SPM) rates in the lagoon (Sfriso et al., 2005a,b) show that high amounts of surface sediments (600-1000 kg dwt m $\left.{ }^{-2} \mathrm{y}^{-1}\right)$ are re-suspended and can re-deposit in situ or spread all around. The SPM can have particularly high nutrient and pollutant concentrations and can spread the contaminants accumulated in "hot areas" over the whole lagoon (Sfriso et al., 2003; Secco et al., 2005; Bernardello et al., 2006). Tapes philippinarum is a sediment-feeder and filter-feeder organism that lives sunken in the sediment. It can assimilate pollutants directly from the sediment or by its filter-feeding activity; however, the relationship between the metal concentration in the surface sediments and in the clam soft tissues is not always clear.

This paper aims at investigating such relationship and also the different role played by the sediment and SPM concentrations. The depuration time of commercial clams harvested in contaminated areas is usually studied in order to reduce clam contamination before the product is sold. In this paper the results of an investigation on seed produced in a clam-farm placed at Marano lagoon after seeding in polluted areas are presented. The growth up to the commercial size in nets placed on the bottom or suspended in the water column was considered.

Finally, the peak concentrations of some toxicants such as $\mathrm{Hg}$, As, $\mathrm{Cd}$ and $\mathrm{Pb}$ in clam tissues have been examined to provide information on how and when these contaminants affect the product and on the risk for human health. 


\section{Materials and methods}

\subsection{Area description}

Sampling sites were selected in two lagoons of the northern Adriatic Sea: Venice and Marano (Fig. 1). Two stations (Fusina and San Giuliano) were selected in the Venice lagoon where free clamharvesting is high and effects on sedimentation rates (Sfriso et al., 2005a,b), benthic macrofauna (Pranovi and Giovanardi, 1994) and public health (Orel et al., 2000) are a serious problem. These stations are characterized by hypertrophic conditions and marked by industrial and urban contamination (Marcomini et al., 1997; Guerzoni and Raccanelli, 2003; Sfriso et al., 2005c). Another station was selected in the Marano lagoon, in a sandy area close to Lignano Sabbiadoro sea-inlet, where the trophic conditions and pollutants, with the exception of Hg, are low (Matassi et al., 2007). That station is close to the clam-farm (ALMAR: Acquacoltura Lagunare Marinetta di Marano lagunare Soc.Coop.Ar.l.) where the seed for the experimental design was produced and reared to $25 \mathrm{~mm}$ (the highest clam-size locally employed for seeding in farming areas).

\subsection{Environmental parameter determination}

During each sampling campaign, some environmental parameters related to the water column (temperature, chlorophyll $a$, and filtered particulate matter), surface sediments (grain-size, density, porosity, moisture, and organic carbon) and settled particulate matter (grain-size and organic carbon) were also measured according to the sampling procedure and the analytical methods reported in Sfriso et al. (2005a,c).

\subsection{Clam production, seeding, and harvesting}

Some tens of kilograms of clam seed were seeded both at Marano lagoon and Fusina and San Giuliano (Venice lagoon). In each site, clam stocks of ca. $2 \mathrm{~kg}$ were put into nylon nets; $4-5$ nets were placed on the bottom surface and $4-5$ nets were suspended at ca. $30 \mathrm{~cm}$ from the bottom by means of polyethylene cages. Seeding occurred in early April 2003. Later, during three progressive sampling periods, two clam sub-samples, one placed on the bottom (B-clams) and the other suspended in the water column (S-clams), were collected together with samples of sediment and SPM. The first sampling period was in October 2003 (first growing stage), the second in June-July 2004 (medium-size clams) and the last in January 2005 (adult clams ready for sale). The sites were inspected each month to clean the nets from macrophytes and sediments and to remove the fouling organisms and predators. After harvesting, clams were carefully cleaned from mud and kept in current seawater near Malamocco port-entrance for $24 \mathrm{~h}$ to eliminate the sediment placed between the valves and to favour the expulsion of the sediment trapped in the digestive apparatus.

\subsection{Clam measurements and sample preparation}

Sixty samples of the initial clam stock (seed) and 60 clam samples per area, exposure and campaign (1140 clams as a total) were weighed and measured (length, width, and thickness) in order to determine their growth progress. Clams were then shelled and the soft tissues were washed with Milli-Q water, dripped for some minutes in a nylon net, weighed and placed in acid-washed glass containers. Samples were freeze-dried, crashed and powered in a rotor speed mill (Pulverisette 14, Fritsch GmbH, Germany) and stored up to metal determination.

\subsection{Sample digestion and metal analyses}

The metals analyzed in surface sediments, SPM and clam tissues were: $\mathrm{Hg}$, As, $\mathrm{Cd}, \mathrm{Pb}, \mathrm{Cr}, \mathrm{Cu}, \mathrm{Co}, \mathrm{Ni}, \mathrm{Zn}, \mathrm{V}, \mathrm{Mn}$, and $\mathrm{Fe}$.

Sample digestion was carried out using reagents of analytical grade. Milli-Q water with a resistivity of $18.2 \mathrm{M} \Omega \mathrm{cm}$ was used for the preparation of reagents and standard solutions. All the containers and the glassware used were soaked for $48 \mathrm{~h}$ in $0.1 \mathrm{M} \mathrm{HNO}_{3}$ and rinsed carefully with Milli-Q water.

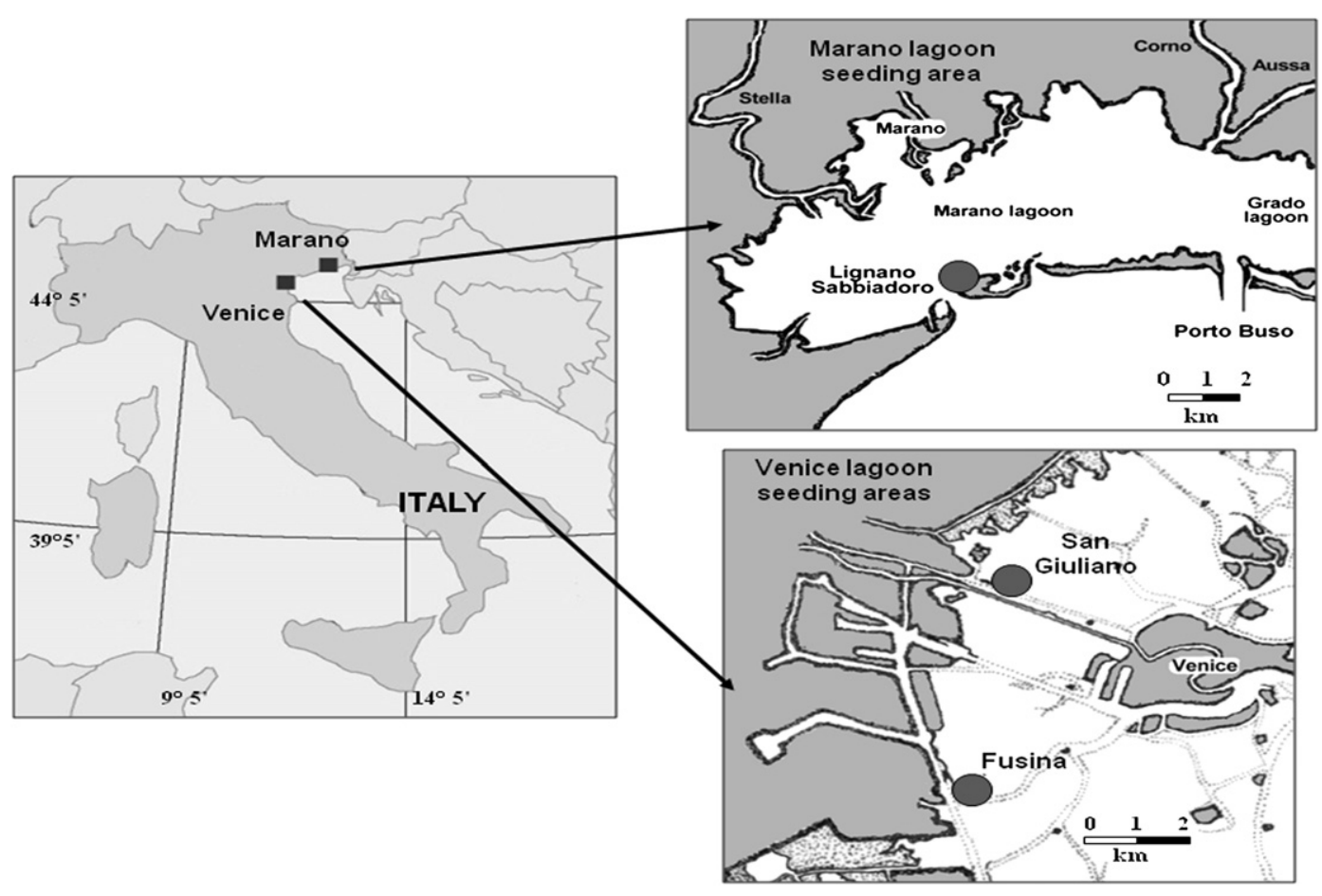

Fig. 1. Sampling sites in Venice and Marano lagoons. 
Aliquots of about $0.3 \mathrm{~g}$ of lyophilized clam samples were microwave-digested in closed Teflon vessels in a CEM MDS-2000 microwave oven, with $8 \mathrm{ml}$ of concentrated $\mathrm{HNO}_{3}, 4 \mathrm{ml}$ of $30 \% \mathrm{H}_{2} \mathrm{O}_{2}$ and $4 \mathrm{ml}$ of $\mathrm{H}_{2} \mathrm{O}$, following a three-step program:

5 min at $T_{\max }=100{ }^{\circ} \mathrm{C}, P_{\max }=80 \mathrm{psi}$, power $=250 \mathrm{~W}$.
$5 \mathrm{~min}$ at $T_{\max }=120^{\circ} \mathrm{C}, P_{\max }=120 \mathrm{psi}$, power $=380 \mathrm{~W}$.
$15 \mathrm{~min}$ at $T_{\max }=170^{\circ} \mathrm{C}, P_{\max }=160 \mathrm{psi}$, power $=570 \mathrm{~W}$.

The resulting digests were made up to volume $(25 \mathrm{ml})$ with MilliQ water, transferred in polyethylene containers and preserved at $4{ }^{\circ} \mathrm{C}$. Aliquots of about $0.1 \mathrm{~g}$ of lyophilized sediment and SPM samples were subjected to microwave digestion with $3 \mathrm{ml}$ of aqua regia $\left(\mathrm{HCl}: \mathrm{HNO}_{3} 3: 1\right), 1.5 \mathrm{ml}$ of $\mathrm{HF}$ and $5 \mathrm{ml}$ of Milli-Q water, in a single step for $60 \mathrm{~min}$ at $T_{\max }=180^{\circ} \mathrm{C}, P_{\max }=140 \mathrm{psi}$, power $=630 \mathrm{~W}$. After cooling, samples were added with $5 \mathrm{ml}$ of a saturated boric acid solution and subjected to a second digestion cycle. The digests were then made up to volume $(50 \mathrm{ml})$ with Milli- $\mathrm{Q} \mathrm{H}_{2} \mathrm{O}$, transferred in polyethylene containers and preserved at $4{ }^{\circ} \mathrm{C}$. These digestion procedures induced the complete solubilization of the soft tissue samples, sediment and SPM, avoiding thereby any necessity of filtration.

Metal analysis was performed with a Perkin-Elmer Optima 3100 XL ICP-AES and an Elan 6100 ICP-MS, depending on the concentration levels. Mercury was determined by CV-AAS with a Varian SpectrAA-250 Plus spectrometer, equipped with a VGA-77 cold vapor generation system. The certified reference materials PACS-2 (marine sediment - National Research Council Canada), DORM-2 (dogfish muscle - National Research Council Canada) and NIST SRM 2976 (mussel tissue - Community Bureau of Reference) were used to validate the analytical methodology and to evaluate accuracy and precision. Recoveries ranged from 91 to $106 \%$, while relative standard deviations of eight replicates were within $7 \%$.

\subsection{Law contamination limits in clams and sediments}

Unfortunately, an international protocol establishing what the toxic concentration limits are for an environment to be considered polluted does not exist and for clams only European reference values for $\mathrm{Hg}\left(0.5 \mu \mathrm{g} \mathrm{g}^{-1} \mathrm{fwt}\right), \mathrm{Cd}\left(1.0 \mu \mathrm{g} \mathrm{g}^{-1} \mathrm{fwt}\right)$ and $\mathrm{Pb}$ $\left(1.5 \mu \mathrm{g} \mathrm{g}^{-1} \mathrm{fwt}\right.$ ) are available (CEE Reg. No. 1881/2006). In the Venice lagoon, however, the sediment contamination has been always considered a serious problem, because canals have to be dredged and the sediments disposed of. For that reason the Italian Ministry of the Environment (1993) produced a protocol in which sediments are divided into three contamination classes $(A, B, C$, where $\mathrm{A}=$ low contamination, $\mathrm{B}=$ moderate contamination and $\mathrm{C}=$ high contamination) on the basis of the concentrations of eight metals and four organic compounds (Table 1). Those values have been compared with the concentrations found in the study areas.

\subsection{Statistical analyses}

All clam metrics and metal concentrations in surface sediments, SPM and clams have been analyzed by one-way ANOVA to highlight differences between stations, periods and clam exposures. Pearson's coefficients were calculated by considering only metals in clams from all the stations and exposures (normal matrix) and metals in sediments, SPM, the clams placed on the sediment surface (S-clams) and suspended in the water column (S-clams) for all the stations together and for each single station (transpose matrix). Finally the principal component analysis (PCA) discriminated the loading of metal associations or single elements in the total metal variance.
Table 1

Environmentally safe criteria to excavate, transport and reuse sediments from the Venice canals (Ministry of Environment, 1993). PAH, polycyclic aromatic hydrocarbons; PCBs, polychlorinated biphenils; DDTs, dichloro-diphenyl-trichloroethane and metabolites; dwt; dry weight

\begin{tabular}{|c|c|c|c|}
\hline \multirow[t]{4}{*}{ Compound } & \multicolumn{3}{|c|}{ Contamination } \\
\hline & Low & Moderate & High \\
\hline & \multicolumn{3}{|c|}{ Class composition } \\
\hline & A & B & C \\
\hline$\overline{\mathrm{Hg}}$ & 0.5 & 2 & 10 \\
\hline $\mathrm{Cd}$ & 1 & 5 & 20 \\
\hline $\mathrm{Pb}$ & 45 & 100 & 500 \\
\hline As & 15 & 25 & 50 \\
\hline $\mathrm{Cr}$ & 20 & 100 & 500 \\
\hline $\mathrm{Cu}$ & 40 & 50 & 400 \\
\hline $\mathrm{Ni}$ & 45 & 50 & 150 \\
\hline $\mathrm{Zn}$ & $\begin{array}{l}200 \\
\left(\mathrm{ng} \mathrm{g}^{-1} \mathrm{dwt}\right)\end{array}$ & 400 & 3000 \\
\hline PAHs & 1000 & 10,000 & 20,000 \\
\hline PCBs & 10 & 200 & 2000 \\
\hline DDTs & 1 & 20 & 500 \\
\hline
\end{tabular}

\section{Results}

\subsection{Environmental parameters}

The values of environmental variables measured in the water column, surface sediments and SPM are shown in Table 2. In particular, water temperature at Fusina was ca. $1-3{ }^{\circ} \mathrm{C}$ higher than in the other stations. This value reached even $8-10^{\circ} \mathrm{C}$ in the canal near the Fusina thermoelectric plant outflow (Socal et al., 1999; Facca et al., 2002a,b).

The grain-size composition of the surface sediments and SPM in the three sites was different. Sediments at San Giuliano had a percentage of fines (particles with diameter $<63 \mu \mathrm{m}$ ) of $96.1 \%$, whereas at Fusina and Marano sediments were sandy (fines: $3.9 \%$ and $7.5 \%$, respectively). Consequently, the amount of sediment per volume unit (dry density) was $0.83 \mathrm{~g} \mathrm{dwt} \mathrm{cm}^{-3}$ at San Giuliano, $1.47 \mathrm{~g} \mathrm{dwt} \mathrm{cm}^{-3}$ at Fusina and $1.43 \mathrm{~g} \mathrm{dwt} \mathrm{cm}^{-3}$ at Marano. The sediment at San Giuliano showed the highest porosity with $69.8 \%$ water content. The water content lowered to $39 \%$ and $48 \%$ at Fusina and Marano, respectively.

As for SPM, the amounts collected by sedimentation traps at San Giuliano and Fusina were 952 and $760 \mathrm{~kg} \mathrm{dwt} \mathrm{m}^{-2} \mathrm{y}^{-1}$, respectively (Sfriso et al., 2005a,b). At Marano, the available data referred only to some periods, therefore in analogy with an area placed near Malamocco inlet in the Venice lagoon where SPM rates are similar to those recorded at Marano station, the annual SPM rate was expected to be ca. $200-300 \mathrm{~kg} \mathrm{dwt} \mathrm{m}^{-2} \mathrm{y}^{-1}$. The amount of particulate matter suspended in the water column and obtained by filtration (FPM) reflected the SPM amounts: $78.8 \mathrm{mg} \mathrm{dwt} \mathrm{L}^{-1}$ at San Giuliano, 57.1 $\mathrm{mg} \mathrm{dwt} \mathrm{L}^{-1}$ at Fusina and $45.1 \mathrm{dwt} \mathrm{mg} \mathrm{L}^{-1}$ at Marano. The percentage of fines in SPM depended on the station location. At San Giuliano SPM grain-size was a bit lower than surface sediments (fines: ca. 93.2\%), whereas at Marano and Fusina the percentage of fine SPM was twice (ca.15.7\%) and three times (ca. 14.4\%) as high as the surface sediments, respectively.

The trophic level of the stations can be inferred by the concentration of organic carbon (Corg) both in surface sediments and in SPM (Table 2). Organic carbon was very low at Marano and Fusina, which are characterized by sandy sediments and rather high at San Giuliano where on average it reached 12.2 and $18.3 \mathrm{mg} \mathrm{g}^{-1} \mathrm{dwt}$ in surface sediments and SPM, respectively.

\subsection{Tapes philippinarum morphometry}

On an average, B-clams reached a bigger size (length, width, thickness) and weight than S-clams (Fig. 2). The difference was 
Table 2

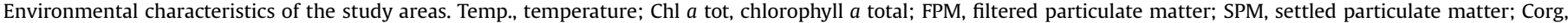
organic carbon; dwt, dry weight; std., standard deviation

\begin{tabular}{|c|c|c|c|c|c|c|c|c|c|c|}
\hline \multirow[t]{3}{*}{ Station } & \multirow[t]{3}{*}{ Date } & \multicolumn{3}{|c|}{ Water column } & \multicolumn{4}{|c|}{ Surface sediment } & \multicolumn{2}{|l|}{ SPM } \\
\hline & & \multirow{2}{*}{$\frac{\text { Temp. }}{\text { Bottom }}$} & \multirow{2}{*}{$\frac{\text { Chl } a \text { tot }}{\mu \mathrm{g} \mathrm{L}^{-1}}$} & \multirow{2}{*}{$\frac{\text { FPM }}{\mathrm{mg} \mathrm{L}^{-1}}$} & \multirow{2}{*}{$\frac{\text { Grain-size }}{\%<63 \mu \mathrm{m}}$} & \multicolumn{2}{|l|}{ Density } & \multirow{2}{*}{$\frac{\text { Corg }}{\mathrm{mg} \mathrm{g}^{-1} \mathrm{dwt}}$} & \multirow{2}{*}{$\frac{\text { Grain-size }}{\%<63 \mu \mathrm{m}}$} & \multirow{2}{*}{$\frac{\text { Corg }}{\mathrm{mg} \mathrm{g}^{-1} \mathrm{dwt}}$} \\
\hline & & & & & & $\mathrm{g} \mathrm{cm}^{-3} \mathrm{dwt}$ & $\mathrm{g} \mathrm{cm}^{-3} \mathrm{fwt}$ & & & \\
\hline \multirow[t]{6}{*}{ Marano } & Seeding & 18.2 & 2.94 & 21.0 & 7.46 & 1.42 & 1.93 & 1.28 & 21.5 & 4.74 \\
\hline & 1st period & 18.0 & 3.64 & 67.4 & 7.19 & 1.43 & 1.92 & 1.24 & 13.7 & 4.90 \\
\hline & 2nd period & 25.0 & 1.33 & 54.0 & 6.82 & 1.41 & 1.90 & 1.32 & 10.2 & 4.53 \\
\hline & 3rd period & 5.9 & 4.98 & 38.0 & 8.51 & 1.45 & 1.94 & 1.43 & 17.5 & 7.83 \\
\hline & Mean & 16.8 & 3.2 & 45.1 & 7.5 & 1.43 & 1.92 & 1.32 & 15.7 & 5.50 \\
\hline & Std. & 7.95 & 1.5 & 20.1 & 0.73 & 0.02 & 0.02 & 0.08 & 4.9 & 1.56 \\
\hline \multirow[t]{6}{*}{ Fusina } & Seeding & 20.1 & 3.85 & 61.8 & 3.89 & 1.50 & 1.90 & 1.57 & 12.6 & 3.32 \\
\hline & 1st period & 18.6 & 15.92 & 54.1 & 4.43 & 1.53 & 1.93 & 1.94 & 19.6 & 3.71 \\
\hline & 2nd period & 23.8 & 15.68 & 60.2 & 4.11 & 1.50 & 1.93 & 1.47 & 18.1 & 3.18 \\
\hline & 3rd period & 9.6 & 1.76 & 52.4 & 3.33 & 1.51 & 1.94 & 1.28 & 7.56 & 3.28 \\
\hline & Mean & 18.0 & 9.3 & 57.1 & 3.9 & 1.51 & 1.93 & 1.56 & 14.4 & 3.37 \\
\hline & Std. & 6.0 & 7.5 & 4.6 & 0.46 & 0.01 & 0.02 & 0.28 & 5.5 & 0.23 \\
\hline \multirow[t]{6}{*}{ San Giuliano } & Seeding & 17.8 & 7.77 & 77.3 & 95.4 & 0.85 & 1.51 & 11.3 & 92.5 & 17.5 \\
\hline & 1st period & 16.7 & 15.32 & 69.2 & 94.9 & 0.88 & 1.60 & 11.6 & 93.7 & 23.6 \\
\hline & 2nd period & 22.0 & 22.80 & 93.3 & 98.3 & 0.80 & 1.49 & 12.5 & 98.0 & 18.5 \\
\hline & 3rd period & 6.0 & 2.72 & 75.4 & 95.8 & 0.80 & 1.47 & 13.3 & 88.6 & 13.5 \\
\hline & Mean & 15.6 & 12.2 & 78.8 & 96.1 & 0.83 & 1.52 & 12.2 & 93.2 & 18.3 \\
\hline & Std. & 6.81 & 8.8 & 10.3 & 1.49 & 0.04 & 0.06 & 0.88 & 3.9 & 4.12 \\
\hline
\end{tabular}

particularly evident at Fusina (one-way ANOVA: length $p<0.01$, width, thickness and weight $p<0.05$ ), which is a hypertrophic area characterized by sandy sediments (Table 2 ), the result of past floods by the River Brenta. Moreover, notwithstanding the coarse sediments, clams harvested at Fusina (Fig. 2b) at the 3rd sampling period reached sizes (length: $38.3 \mathrm{~mm}$, width: $29.2 \mathrm{~mm}$, thickness: $19.9 \mathrm{~mm})$ and weight $(14.9 \mathrm{~g})$ higher than in the other stations (one-way ANOVA: length $p<3.5 \times 10^{-24}$, width $p<1.0 \times 10^{-24}$, thickness $p<4.5 \times 10^{-5}$ and weight $\left.p<9.0 \times 10^{-11}\right)$. In that area clams were little affected by predators and showed a negligible mortality.

As expected, the lowest clam growth was recorded at Marano (Fig. 2a) where the mean weight was only $11.6 \mathrm{~g}$ at the end of the 3rd sampling period. In that area clams were predated by the gastropod Hexaplex trunculus, which killed ca. $30-40 \%$ of the exposed samples. At San Giuliano clam-size and weight were intermediate. In that area, about $50 \%$ of the samples were killed by the gastropod Nassarius nitidus, and the overgrowth on the clam
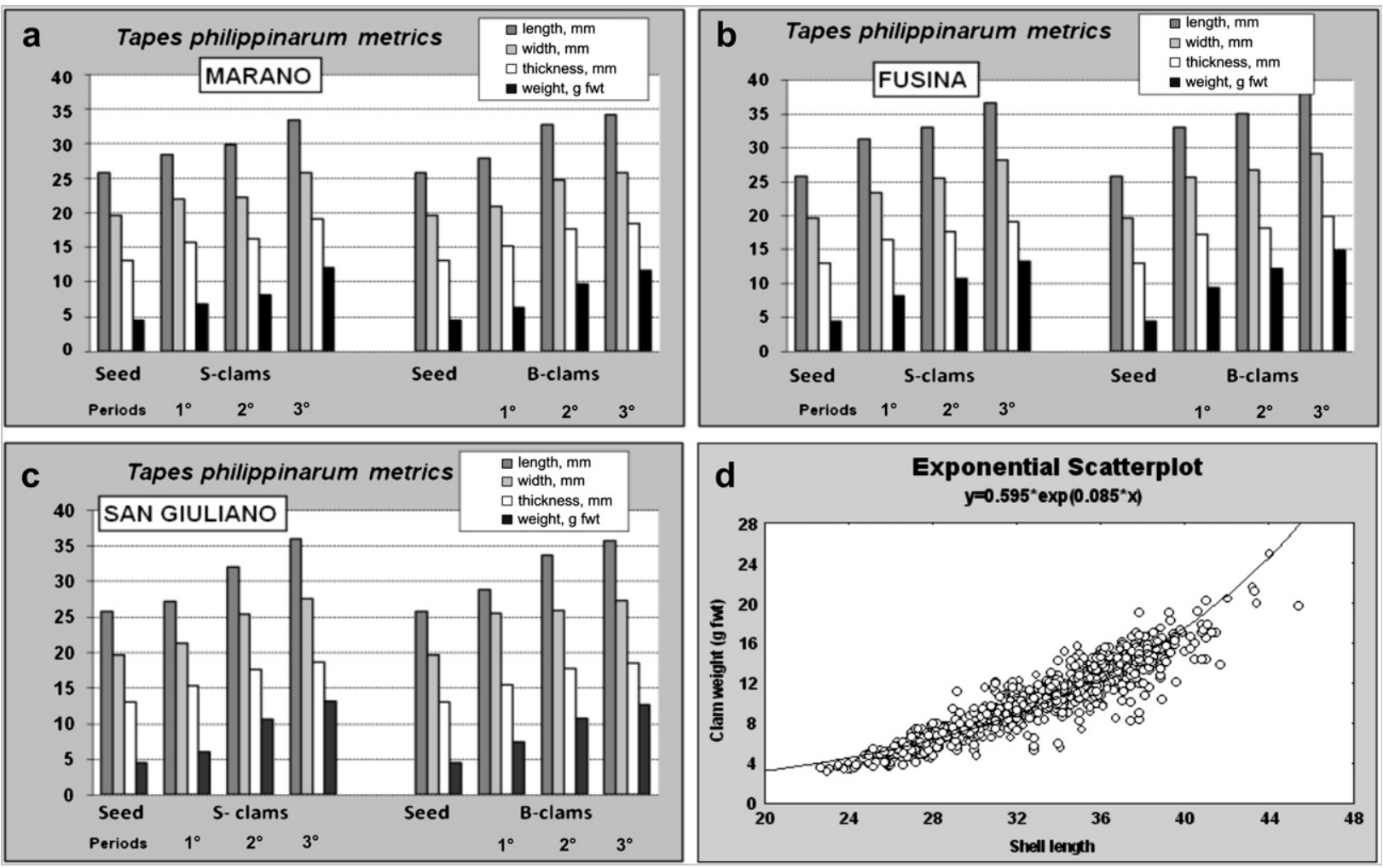

Fig. 2. Seed and clam measurements during the three sampling periods in the study areas and exponential scatter plot between shell length and clam weight. 
shells of the bivalve Cassostrea gigas, the crustacean Balanus sp. and many calcareous worms belonging to Serpulidae. The scatter plot between shell length and clam weight of all clam shells fitted with an exponential trend (Fig. 2d).

\subsection{Metal concentrations}

The concentrations of the 12 metals out of which $\mathrm{As}, \mathrm{Hg}, \mathrm{Cd}, \mathrm{Pb}$ and $\mathrm{Cr}$ are elements, which exhibit high toxicity both for biota and for man, are shown in Table 3. In general, SPM showed concentrations higher than in surface sediments, but metals showed different behaviors and not all of them were bio-concentrated by clams. Moreover, it must be considered that the regulatory reference limits, when available, refer to clam wet weight and the ratio dry/wet weight of the samples ranged from 0.146 to $0.195 \%$ (Table 3).

\subsubsection{Mercury ( $\mathrm{Hg})$}

Mercury showed relatively high concentrations in the surface sediments and SPM of both Venice and Marano lagoons. The most contaminated area was San Giuliano where the sediments showed concentrations ranging between 0.99 and $1.22 \mu \mathrm{g} \mathrm{g}^{-1} \mathrm{dwt}$ (Class B), whereas in SPM Hg concentrations doubled (1.24-2.28 $\mu \mathrm{g} \mathrm{g}^{-1} \mathrm{dwt}$ ) in two samples out of three. At Marano sediment concentrations were about half (ca. $0.40-0.57 \mu \mathrm{g} \mathrm{g}^{-1} \mathrm{dwt}$, Class B) as high as at San Giuliano. In SPM the highest value was $1.17 \mu \mathrm{g} \mathrm{g}^{-1} \mathrm{dwt}$. At Fusina $\mathrm{Hg}$ concentrations both in the surface sediments and SPM were low (0.10-0.22 $\mu \mathrm{g} \mathrm{g}^{-1} \mathrm{dwt}$, Class A) with exception of SPM where $\mathrm{Hg}$ peaked up to $0.66 \mu \mathrm{g} \mathrm{g}^{-1} \mathrm{dwt}$ during the 1 st campaign.

In clams the highest concentrations were recorded at Marano with $1.35-2.03 \mu \mathrm{g} \mathrm{g}^{-1} \mathrm{dwt}$ corresponding to $0.25-0.30 \mu \mathrm{g} \mathrm{g}^{-1} \mathrm{fwt}$, showing a significant increase in comparison with seed (one-way ANOVA: $p<3.0 \times 10^{4}$ ). At San Giuliano and Fusina clams showed quite similar concentrations, which were significantly lower than seed (one-way ANOVA: San Giuliano $p<4.0 \times 10^{9}$, Fusina $\left.p<2.0 \times 10^{9}\right)$ ranging from 0.18 to $0.42 \mu \mathrm{g} \mathrm{g}^{-1} \mathrm{dwt}(0.03$ to $0.07 \mu \mathrm{g} \mathrm{g}^{-1}$ fwt). No significant difference was found by comparing B-clams with S-clams.

\subsubsection{Arsenic (As)}

Arsenic showed relatively high concentrations in the surface sediments (8.7-17.1 $\mathrm{\mu g} \mathrm{g}^{-1} \mathrm{dwt}$, Class B) and SPM (12.6$\left.22.9 \mu \mathrm{g} \mathrm{g}^{-1} \mathrm{dwt}\right)$ at San Giuliano whereas at Marano the sediment (4.0-6.6 $\mu \mathrm{g} \mathrm{g}^{-1} \mathrm{dwt}$, Class A) and SPM concentrations (7.4$8.7 \mu \mathrm{g} \mathrm{g}^{-1} \mathrm{dwt}$ ) were significantly lower. At Fusina As concentrations were intermediate (sediment: $7.1-11.8 \mu \mathrm{g} \mathrm{g}^{-1} \mathrm{dwt}$, Class A; SPM: $4.9-8.8 \mu \mathrm{g} \mathrm{g}^{-1} \mathrm{dwt}$ ).

Seed exhibited a particularly high $\left(34.1 \mu \mathrm{g} \mathrm{g}^{-1} \mathrm{dwt}\right)$ concentration. After seeding, at Marano As in clams increased markedly (42.8-64.0 $\mu \mathrm{g} \mathrm{g}^{-1} \mathrm{dwt}$, one-way ANOVA: $\left.p<0.003\right)$. In contrast metal concentrations in clams exposed at Fusina (18.9$30.7 \mu \mathrm{g} \mathrm{g}^{-1} \mathrm{dwt}$ one-way ANOVA: $\left.p<0.002\right)$ and San Giuliano (18.9-28.2 $\mu \mathrm{g} \mathrm{g}^{-1}$ fwt, one-way ANOVA: $p<2 \times 10^{5}$ ) exhibited As concentrations lower than in seed. No significant difference was found by comparing As concentrations in B-clams with concentrations in S-clams.

\subsubsection{Cadmium (Cd)}

Cadmium concentration was high in the sediments (0.99$1.43 \mu \mathrm{g} \mathrm{g}^{-1} \mathrm{dwt}$, Class B) and SPM (1.48-1.90 $\left.\mu \mathrm{g} \mathrm{g}^{-1} \mathrm{dwt}\right)$ at San Giuliano. At Marano and Fusina, Cd concentrations were markedly lower, ranging between 0.09 and $0.62 \mu \mathrm{g} \mathrm{g}^{-1} \mathrm{dwt}$ (Class A).

Clams showed values ranging between 0.87 and $1.38 \mu \mathrm{g} \mathrm{g}^{-1} \mathrm{dwt}$ at San Giuliano, $0.76-2.19 \mu \mathrm{gg}^{-1} \mathrm{dwt}$ at Fusina and 0.26$0.49 \mu \mathrm{g} \mathrm{g}^{-1} \mathrm{dwt}$ at Marano. The cadmium increase was significant in all the stations (one-way ANOVA: Marano $p<2.7 \times 10^{3}$, Fusina $p<8.3 \times 10^{4}$, San Giuliano $p<8.5 \times 10^{7}$ ) and in particular in the Venice stations. In the B-clams at Fusina during the 3rd period Cd concentrations were about one order of magnitude higher than in the seed and 6-8 times higher than in the sediment and SPM. No significant difference between S-clam and B-clam concentrations was recorded.

\subsubsection{Chromium (Cr)}

The highest concentrations were recorded in the sediments at Fusina. The surface sediments showed values ranging between 58 and $95 \mu \mathrm{g} \mathrm{g}^{-1} \mathrm{dwt}$ (Class B) and in SPM Cr peaked up to $174 \mu \mathrm{g} \mathrm{g}^{-1} \mathrm{dwt}$. At San Giuliano and Marano sediment and SPM concentrations were similar (San Giuliano: $41-60 \mu \mathrm{g} \mathrm{g}^{-1} \mathrm{dwt}$, Class B; Marano: $39-70 \mu \mathrm{g} \mathrm{g}^{-1} \mathrm{dwt}$, Class B).

In clams $\mathrm{Cr}$ ranged between 1.93 and $4.03 \mu \mathrm{g} \mathrm{g}^{-1} \mathrm{dwt}$ at San Giuliano and Fusina, and between 3.10 and $5.70 \mu \mathrm{g} \mathrm{g}^{-1} \mathrm{dwt}$, at Marano. In comparison with seed concentrations, the increase of $\mathrm{Cr}$ in clam tissues was not significant at Marano whereas its concentrations decreased significantly at Fusina (one-way ANOVA: $p<3.4 \times 10^{4}$ ) and at San Giuliano (one-way ANOVA: $p<1.7 \times 10^{3}$ ). Therefore, there was no direct relationship between $\mathrm{Cr}$ concentrations in clams, sediment and SPM. Moreover no significant difference was found between S-clams and B-clams.

\subsubsection{Lead $(\mathrm{Pb})$}

Like mercury, $\mathrm{Pb}$ showed the highest concentrations in the sediments (29.6-47.3 $\mu \mathrm{g} \mathrm{g}^{-1} \mathrm{dwt}$, Class B) and SPM (32.5$57.1 \mu \mathrm{g} \mathrm{g}^{-1} \mathrm{dwt}$ ) collected at San Giuliano. At Marano Pb concentrations were low both in the sediments and SPM $\left(2.5-6.3 \mu \mathrm{g} \mathrm{g}^{-1} \mathrm{dwt}\right.$, Class A), whereas at Fusina the concentrations in the sediments (8.4-12.9 $\mu \mathrm{g} \mathrm{g}^{-1} \mathrm{dwt}$, Class A) and SPM (10.3-22.2 $\left.\mu \mathrm{g} \mathrm{g}^{-1} \mathrm{dwt}\right)$ showed average levels.

In the clams of the three stations $\mathrm{Pb}$ concentrations were lower than $2.54 \mu \mathrm{g} \mathrm{g}^{-1} \mathrm{dwt}$ corresponding to $0.45 \mu \mathrm{g} \mathrm{g}^{-1} \mathrm{fwt}$, without significant differences between S-clams and B-clams. Conversely, a concentration increase was significant (one-way ANOVA: Marano $p<0.04$, Fusina $p<9.0 \times 10^{4}$, San Giuliano $p<9.1 \times 10^{4}$ ) after seeding.

\subsubsection{Copper (Cu)}

As most of the other metals $\mathrm{Cu}$ exhibited the highest concentrations at San Giuliano. Cu ranged between 29 and $43 \mu \mathrm{g} \mathrm{g}^{-1} \mathrm{dwt}$ (Class B) in the surface sediments and between 35 and $51 \mu \mathrm{g} \mathrm{g}^{-1} \mathrm{dwt}$ in SPM. At Marano, Cu concentrations were low in both sediments (2.7-4.3 $\mu \mathrm{g} \mathrm{g}^{-1} \mathrm{dwt}$, Class A) and SPM (5.8$11.3 \mu \mathrm{g} \mathrm{g}^{-1} \mathrm{dwt}$ ). Fusina showed concentrations similar to Marano (sediments: 5.7-7.7 $\mu \mathrm{g} \mathrm{g}^{-1} \mathrm{dwt}$, Class A; SPM: 8.7-15.2 $\mu \mathrm{g} \mathrm{g}^{-1} \mathrm{dwt}$ ).

In clams $\mathrm{Cu}$ concentrations were low. On average $\mathrm{Cu}$ ranged between 8.18 and $13.7 \mu \mathrm{g} \mathrm{g}^{-1} \mathrm{dwt}$ at Marano, 9.79 and $29.0 \mu \mathrm{g} \mathrm{g}^{-1} \mathrm{dwt}$ at Fusina, 10.3 and $15.8 \mu \mathrm{g} \mathrm{g}^{-1} \mathrm{dwt}$ at San Giuliano with significant changes (one-way ANOVA: Marano $p<3.3 \times 10^{4}$, Fusina $p<2.2 \times 10^{3}$, San Giuliano $p<1.0 \times 10^{4}$ ) although trends were not linked to growth. No difference between S-clams and Bclams was recorded except at Fusina where Cu concentrations were higher in B-clams during the 1st and the 3rd periods.

\subsubsection{Nickel (Ni)}

This element had a behavior similar to $\mathrm{Cr}$. The concentrations of $\mathrm{Ni}$ were relatively high with peaks at Fusina (sediments: 34-59 $\mu \mathrm{g} \mathrm{g}^{-1} \mathrm{dwt}$, Class C; SPM: $62-83 \mu \mathrm{g} \mathrm{g}^{-1} \mathrm{dwt}$ ). Rather high concentrations were found also at San Giuliano (sediments: 28$53 \mu \mathrm{gg}^{-1} \mathrm{dwt}$, Class C; SPM 35-51 $\mu \mathrm{gg}^{-1} \mathrm{dwt}$ ). At Marano $\mathrm{Ni}$ showed low concentrations both in the sediments (18$26 \mu \mathrm{g} \mathrm{g}^{-1} \mathrm{dwt}$, Class A) and SPM (17-46 $\mu \mathrm{g} \mathrm{g}^{-1} \mathrm{dwt}$ ).

Although the concentrations in the environment were high, in the clams exposed in the Venice lagoon Ni was not bio-accumulated. 
Table 3

Metals in sediments, SPM and clams. S-clams, suspended clams; B-clams, bottom clams; SPM, settled particulate matter; d.l., detection limit; dwt, dry weight

\begin{tabular}{|c|c|c|c|c|c|c|c|c|c|c|c|c|c|c|}
\hline & & & $\begin{array}{l}\text { As } \\
\left(\mu \mathrm{g} \mathrm{g}^{-1} \mathrm{dwt}\right)\end{array}$ & $\begin{array}{l}\mathrm{Cd} \\
\left(\mu \mathrm{g} \mathrm{g}^{-1} \mathrm{dwt}\right)\end{array}$ & $\begin{array}{l}\mathrm{Cr} \\
\left(\mu \mathrm{g} \mathrm{g}^{-1} \mathrm{dwt}\right)\end{array}$ & $\begin{array}{l}\text { Co } \\
\left(\mu \mathrm{g} \mathrm{g}^{-1} \mathrm{dwt}\right)\end{array}$ & $\begin{array}{l}\mathrm{Cu} \\
\left(\mu \mathrm{g} \mathrm{g}^{-1} \mathrm{dwt}\right)\end{array}$ & $\begin{array}{l}\mathrm{Pb} \\
\left(\mu \mathrm{g} \mathrm{g}^{-1} \mathrm{dwt}\right)\end{array}$ & $\begin{array}{l}\mathrm{Mn} \\
\left(\mu \mathrm{gg}^{-1} \mathrm{dwt}\right)\end{array}$ & $\begin{array}{l}\mathrm{Ni} \\
\left(\mu \mathrm{g} \mathrm{g}^{-1} \mathrm{dwt}\right)\end{array}$ & $\begin{array}{l}\mathrm{V} \\
\left(\mu \mathrm{gg}^{-1} \mathrm{dwt}\right)\end{array}$ & $\begin{array}{l}\mathrm{Zn} \\
\left(\mu \mathrm{g} \mathrm{g}^{-1} \mathrm{dwt}\right)\end{array}$ & $\begin{array}{l}\mathrm{Fe} \\
\left(\mu \mathrm{g} \mathrm{g}^{-1} \mathrm{dwt}\right)\end{array}$ & $\begin{array}{l}\mathrm{Hg} \\
\left(\mu g^{-1} \mathrm{dwt}\right)\end{array}$ \\
\hline Clam seed & & Marano & 34.1 & 0.23 & 4.0 & 1.05 & 8.69 & 0.44 & 12.7 & 5.87 & $<$ d.l. & 76.3 & 444 & 1.05 \\
\hline \multirow[t]{12}{*}{ Marano } & S-clams & 1st Period & 40.4 & 0.40 & 4.52 & 1.58 & 10.5 & 1.41 & 23.0 & 6.29 & 2.68 & 68.7 & 1122 & 1.78 \\
\hline & B-clams & & 45.0 & 0.26 & 4.28 & 2.12 & 11.7 & 1.22 & 25.8 & 7.37 & 3.09 & 74.1 & 1299 & 1.78 \\
\hline & Sediment & & 4.0 & 0.10 & 50.5 & $<$ d.l. & 4.21 & 4.99 & 357 & 23.5 & 16.8 & 18.0 & 5062 & 0.40 \\
\hline & SPM & & 8.7 & 0.09 & 39.4 & $<$ d.l. & 4.76 & 3.38 & 377 & 17.4 & 15.7 & 4.70 & 5744 & 0.47 \\
\hline & S-clams & 2nd Period & 45.8 & 0.35 & 5.70 & 1.36 & 8.18 & 0.62 & 22.4 & 3.6 & 2.32 & 60.0 & 939 & 1.35 \\
\hline & B-clams & & 42.8 & 0.29 & 3.10 & 1.54 & 8.35 & 0.49 & 21.0 & 3.04 & 2.15 & 68.7 & 882 & 1.42 \\
\hline & Sediment & & 6.6 & 0.58 & 53.6 & $<$ d.l. & 3.37 & 6.84 & 386 & 26.3 & 12.0 & 4.10 & 4180 & 0.57 \\
\hline & SPM & & 7.4 & 0.14 & 46.44 & $<$ d.l. & 5.29 & 4.15 & 375 & 28.5 & 17.7 & 11.9 & 6245 & 0.44 \\
\hline & S-clams & 3rd Period & 47.1 & 0.49 & 4.92 & 2.16 & 10.2 & 0.61 & 17.8 & 6.53 & 2.05 & 75.4 & 966 & 1.44 \\
\hline & B-clams & & 64.0 & 0.41 & 4.35 & 2.68 & 13.7 & 0.55 & 13.1 & 8.37 & 1.87 & 81.7 & 913 & 2.03 \\
\hline & Sediment & & 6.6 & 0.10 & 32.2 & $<$ d.l. & 2.73 & 2.44 & 371 & 18.6 & 13.6 & 26.7 & 5074 & 0.55 \\
\hline & SPM & & 8.6 & 0.11 & 69.9 & $<$ d.l. & 11.3 & 6.34 & 389 & 46.5 & 29.5 & 62.0 & 11,324 & 1.17 \\
\hline \multirow[t]{12}{*}{ Fusina } & S-clams & 1st Period & 27.0 & 0.99 & 2.22 & 1.99 & 9.79 & 1.62 & 14.9 & 5.82 & $<$ d.l. & 92.0 & 624 & 0.37 \\
\hline & B-clams & & 22.1 & 1.17 & 3.11 & 1.67 & 29 & 1.83 & 11.9 & 4.05 & $<$ d.l. & 90.3 & 662 & 0.34 \\
\hline & Sediment & & 11.8 & 0.40 & 58.4 & 6.6 & 7.6 & 12.9 & 244 & 34.3 & 22.8 & 83.0 & 10,793 & 0.13 \\
\hline & SPM & & 8.8 & 0.58 & 116 & 6.8 & 15.2 & 22.1 & 293 & 62.4 & 29.0 & 111 & 13,744 & 0.66 \\
\hline & S-clams & 2nd Period & 27.4 & 0.95 & 1.93 & 1.34 & 8.8 & 1.19 & 17.8 & 0.93 & $<$ d.l. & 105.4 & 607 & 0.39 \\
\hline & B-clams & & 18.9 & 0.76 & 1.97 & 0.78 & 13.2 & 0.96 & 8.45 & 0.15 & $<$ d.l. & 90.4 & 239 & 0.18 \\
\hline & Sediment & & 7.10 & 0.43 & 86.19 & 6.73 & 7.46 & 12.72 & 260 & 59.1 & 21.2 & 49.4 & 10,319 & 0.10 \\
\hline & SPM & & 8.60 & 0.62 & 132.6 & 7.91 & 14.25 & 22.18 & 261 & 70.2 & 27.7 & 90.9 & 13,602 & 0.28 \\
\hline & S-clams & 3rd Period & 30.7 & 1.76 & 2.63 & 1.75 & 18.4 & 1.15 & 10.5 & 3.92 & $<$ d.l. & 105 & 607 & 0.40 \\
\hline & B-clams & & 30.7 & 2.19 & 3.56 & 2.38 & 13.3 & 2.54 & 19.0 & 5.20 & $<$ d.l. & 111 & 1079 & 0.41 \\
\hline & Sediment & & 8.5 & 0.28 & 95.4 & 7.17 & 5.69 & 8.37 & 263 & 52.0 & 18.6 & 104 & 14,075 & 0.22 \\
\hline & SPM & & 4.9 & 0.34 & 174 & 6.7 & 8.71 & 10.3 & 236 & 83.0 & 18.0 & 91 & 10,690 & 0.16 \\
\hline \multirow[t]{12}{*}{ San Giuliano } & S-clams & 1st Period & 22.4 & 0.94 & 2.26 & 2.45 & 10.5 & 1.3 & 29.1 & 2.22 & $<$ d.l. & 94.6 & 579 & 0.23 \\
\hline & B-clams & & 26.2 & 0.98 & 3.01 & 2.07 & 10.4 & 1.77 & 18.5 & 3.10 & $<$ d.l. & 102 & 672 & 0.27 \\
\hline & Sediment & & 8.7 & 1.20 & 50.2 & 13.6 & 39.8 & 40.5 & 333 & 27.9 & 53.0 & 272 & 19,222 & 0.99 \\
\hline & SPM & & 22.9 & 1.90 & 58.5 & 15.6 & 51.5 & 57.1 & 410 & 36.5 & 58.5 & 411 & 23,067 & 2.18 \\
\hline & S-clams & 2nd Period & 18.9 & 0.87 & 2.47 & 1.35 & 10.29 & 1.2 & 20.4 & 2.28 & $<$ d.l. & 84.4 & 544 & 0.19 \\
\hline & B-clams & & 22.3 & 0.95 & 1.89 & 1.15 & 15.79 & 0.62 & 10.3 & 2.5 & $<$ d.l. & 110 & 367 & 0.18 \\
\hline & Sediment & & 16.1 & 1.43 & 57.4 & 13.4 & 42.94 & 47.28 & 360 & 53.2 & 57.8 & 263 & 20,720 & 0.91 \\
\hline & SPM & & 18.5 & 1.48 & 60.1 & 13.7 & 44.64 & 50.41 & 425 & 50.7 & 63.6 & 261 & 23,446 & 1.24 \\
\hline & S-clams & 3rd Period & 25.1 & 1.02 & 4.03 & 1.82 & 12.1 & 1.32 & 16.7 & 4.79 & $<$ d.l. & 122 & 714 & 0.38 \\
\hline & B-clams & & 28.2 & 1.38 & 2.05 & 1.73 & 11.9 & 0.92 & 8.04 & 3.69 & $<$ d.l. & 100 & 380 & 0.42 \\
\hline & Sediment & & 17.1 & 0.99 & 41.0 & 13.8 & 29.2 & 29.6 & 347 & 27.3 & 51.9 & 319 & 21,070 & 1.22 \\
\hline & SPM & & 12.6 & 1.39 & 49.6 & 12.7 & 35.4 & 32.5 & 373 & 35.4 & 50.0 & 330 & 21,778 & 2.28 \\
\hline
\end{tabular}


The highest Ni concentration $\left(8.37 \mu \mathrm{g} \mathrm{g}^{-1} \mathrm{dwt}\right)$ was found at Marano where the increase was significant (one-way ANOVA: $p<4.8 \times 10^{5}$ ), whereas in the Venice stations the concentrations during clam growth decreased.

\subsubsection{Zinc $(Z n)$}

This element exhibited the highest concentrations both in the sediments and SPM (263-330 $\mu \mathrm{g} \mathrm{g}^{-1} \mathrm{dwt}$, Class B) at San Giuliano whereas at Marano concentrations were low (sediments: 4$27 \mu \mathrm{g} \mathrm{g}^{-1} \mathrm{dwt}$, Class A; SPM: 5-62 $\mu \mathrm{g} \mathrm{g}^{-1} \mathrm{dwt}$ ). Fusina exhibited intermediate values (sediments: $49-104 \mu \mathrm{g} \mathrm{g}^{-1} \mathrm{dwt}$, SPM 91$\left.111 \mu \mathrm{g} \mathrm{g}^{-1}\right)$.

At Marano $\mathrm{Zn}$ in clam tissues ranged between 60.0 and $81.7 \mu \mathrm{g} \mathrm{g}^{-1} \mathrm{dwt}$. At Fusina and San Giuliano Zn concentrations were higher: 90-111 and 84.4-122 $\mu \mathrm{g} \mathrm{g}^{-1} \mathrm{dwt}$, respectively. The increase of this element was significant only at Fusina (one-way ANOVA: $p<1.2 \times 10^{4}$ ) and at San Giuliano (one-way ANOVA: $p<6.3 \times 10^{4}$ ). No marked difference between S-clams and B-clams was recorded.

\subsubsection{Manganese (Mn)}

Manganese exhibited the highest concentrations both in the sediments (244-386 $\left.\mu \mathrm{g} \mathrm{g}^{-1} \mathrm{dwt}\right)$ and SPM (236-425 $\left.\mu \mathrm{g} \mathrm{g}^{-1} \mathrm{dwt}\right)$ from all the three study areas.

The clams at Fusina showed concentrations (8.45$19.0 \mu \mathrm{g} \mathrm{g}^{-1} \mathrm{fwt}$ ) lower than at San Giuliano (8.04-29.1 $\left.\mu \mathrm{g} \mathrm{g}^{-1} \mathrm{fwt}\right)$ and Marano (13.1-25.8 $\left.\mu \mathrm{g} \mathrm{g}^{-1} \mathrm{fwt}\right)$. The highest clam bioaccumulation (one-way ANOVA: $p<1.6 \times 10^{3}$ ) was in Marano, especially during the 1 st growth period, though later $\mathrm{Mn}$ concentration began to decrease. Similar results were found at San Giuliano. In general, except at Fusina during the 3rd period, Mn seemed to accumulate particularly in S-clams even if the difference was not statistically significant.

\subsubsection{Iron (Fe)}

Iron ranged from high concentrations, both in sediments $\left(10,320-21,070 \mu \mathrm{g} \mathrm{g}^{-1} \mathrm{dwt}\right)$ and SPM $\left(10,690-23,446 \mu \mathrm{g} \mathrm{g}^{-1} \mathrm{dwt}\right)$ in Venice areas, to low concentrations at Marano (range: 4180$11,324 \mu \mathrm{g} \mathrm{g}^{-1} \mathrm{dwt}$ ). However, clam Fe concentrations (ca. 882-

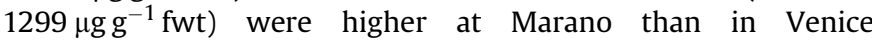
(239-1079 $\left.\mu \mathrm{g} \mathrm{g}^{-1} \mathrm{fwt}\right)$ where bioaccumulation was also highly significant (one-way ANOVA: $p<5.0 \times 10^{6}$ ). No clear difference between B-clams and S-clams was found.

\subsubsection{Cobalt (Co)}

This element showed values below the detection limit at Marano and concentrations ranging between 6.6 and $17.7 \mu \mathrm{g} \mathrm{g}^{-1} \mathrm{dwt}$ in the sediments and SPM at San Giuliano and Fusina. No significant difference between concentrations in sediments and SPM was found.

Concentrations of Co in clams were significantly higher than in seed (one-way ANOVA: Marano $p<1.8 \times 10^{3}$; Fusina $3.1 \times 10^{2}$, San Giuliano $4.2 \times 10^{3}$ ) ranging between 0.78 and $2.68 \mu \mathrm{g} \mathrm{g}^{-1} \mathrm{fwt}$, and no significant difference was found by comparing stations and exposures.

\subsubsection{Vanadium (V)}

The highest sediment concentrations of $\mathrm{V}$ were found at San Giuliano (51.9-57.8 $\mu \mathrm{g} \mathrm{g}^{-1} \mathrm{dwt}$ ). They were lower at Fusina (18.6$22.8 \mu \mathrm{g} \mathrm{g}^{-1} \mathrm{dwt}$ ) and Marano (12-16.8 $\mu \mathrm{g} \mathrm{g}^{-1} \mathrm{dwt}$ ). In SPM the concentrations of $\mathrm{V}$ were a little higher than those found in the sediments.

In the seed and clams from the Venice lagoon $\mathrm{V}$ concentrations were all below the detection limit, whereas at Marano $\mathrm{V}$ concentrations increased significantly (one-way ANOVA: $1.6 \times 10^{7}$ ) ranging between 1.87 and $3.09 \mu \mathrm{g} \mathrm{g}^{-1}$ fwt. No significant difference was found by comparing clam exposures.

\subsection{Statistical analyses}

The Pearson's coefficients of all metals in clam tissues are displayed in Table 4. Mercury, As and $\mathrm{Cr}$ appear significantly correlated with each other and with some other elements such as $\mathrm{Ni}, \mathrm{V}, \mathrm{Zn}$, and Fe whereas $\mathrm{Cd}$ was correlated only with $\mathrm{Zn}$. Vanadium displays significant correlations with $\mathrm{Zn}$ and Fe.

By considering metals in clams, surface sediment and SPM in the three stations both S-clam and B-clam concentrations are more highly correlated to SPM concentrations than to the surface sediment (Table 5). However, by considering each station separately, results are different. In fact, at Marano, metals in S-clams and Bclams are highly correlated to sediment concentrations. At Fusina, B-clams are on average highly correlated to sediment concentrations and S-clams to SPM. At San Giuliano no difference was found.

The highest number of significant correlations was found for $\mathrm{Cd}$ (Table 6). In general significant correlations were found between Sclams and B-clams $(p<0.001)$ as well as between sediment and SPM $(p<0.001)$ but not between clams and inorganic matrices. By considering each station a significant correlation between $\mathrm{Cd}$ in Sclams and sediment $(p<0.05)$ was recorded at San Giuliano.

Neglecting the significant correlations between B-clams and Sclams and between surface sediments and SPM recorded by considering both all the stations together and every single station (Table 6), significant correlations between clams and the inorganic matrices were recorded only for $\mathrm{Hg}, \mathrm{Cd}, \mathrm{As}, \mathrm{Zn}$ and $\mathrm{Fe}$. In particular, As, $\mathrm{Zn}$ and Fe concentrations in S-clams were significantly correlated to SPM values, whereas $\mathrm{Hg}$ and $\mathrm{Cd}$ in S-clams were significantly correlated to the values recorded in the surface sediments.

The PCA analysis shows that $57.6 \%$ of the whole variance system (Table 7) is explained by the first component which enhances the significant loading of $\mathrm{Co}, \mathrm{Cu}, \mathrm{Pb}, \mathrm{Mn}, \mathrm{Ni}, \mathrm{V}, \mathrm{Zn}$, and $\mathrm{Fe}$. The second component, with $24.3 \%$ of the total variance is characterized by the significant loading of As, whereas the third component, with a variance of $6 \%$, enhances the significant loading of $\mathrm{Hg}$.

\section{Discussion and conclusions}

The three study areas are quite different from the morphological, grain-size and trophic point of view but, in general, clam growth was probably mainly affected by water temperature that, on average, was higher at Fusina, especially in winter (Table 2). That area receives the cooling waters from a thermoelectric plant (Socal et al., 1999; Facca et al., 2002a,b) and clams grow bigger and more rapidly than anywhere else in the lagoon. However, clam-fishing and clam-farming in that area are forbidden (Orel et al., 2000), because water and sediments are highly contaminated (Marcomini et al., 1997; Bellucci et al., 2000, 2002). Nevertheless, recent studies (Secco et al., 2005; Sfriso et al., 2005c; Bernardello et al., 2006) have provided evidence that the nutrients and contaminants recorded in that area have recently decreased significantly.

\section{Table 4}

Correlation matrix between metals in clams. $P<0.001, r<|0.73|$

\begin{tabular}{lrrrlllllllll}
\hline & \multicolumn{1}{c}{ As } & $\mathrm{Cd}$ & $\mathrm{Cr}$ & $\mathrm{Co}$ & $\mathrm{Cu}$ & $\mathrm{Pb}$ & $\mathrm{Mn}$ & $\mathrm{Ni}$ & $\mathrm{V}$ & $\mathrm{Zn}$ & $\mathrm{Fe}$ & $\mathrm{Hg}$ \\
\hline $\mathrm{As}$ & 1 & & & & & & & & & & & \\
$\mathrm{Cd}$ & -0.51 & 1 & & & & & & & & & & \\
$\mathrm{Cr}$ & 0.73 & -0.46 & 1 & & & & & & & & & \\
$\mathrm{Co}$ & 0.42 & 0.21 & 0.25 & 1 & & & & & & & & \\
$\mathrm{Cu}$ & -0.24 & 0.42 & -0.18 & 0.04 & 1 & & & & & & & \\
$\mathrm{~Pb}$ & -0.42 & 0.68 & -0.18 & 0.36 & 0.32 & 1 & & & & & & \\
$\mathrm{Mn}$ & 0.21 & -0.31 & 0.37 & 0.37 & -0.44 & 0.17 & 1 & & & & & \\
$\mathrm{Ni}$ & 0.72 & -0.26 & 0.66 & 0.56 & 0.01 & -0.02 & 0.09 & 1 & & & & \\
$\mathrm{~V}$ & 0.81 & -0.66 & 0.73 & 0.20 & -0.27 & -0.34 & 0.49 & 0.54 & 1 & & & \\
$\mathrm{Zn}$ & -0.60 & 0.76 & -0.57 & 0.11 & 0.29 & 0.47 & -0.37 & -0.32 & -0.76 & 1 & & \\
$\mathrm{Fe}$ & 0.68 & -0.22 & 0.73 & 0.54 & -0.14 & 0.21 & 0.63 & 0.65 & 0.79 & -0.42 & 1 & \\
$\mathrm{Hg}$ & 0.93 & -0.67 & 0.77 & 0.27 & -0.26 & -0.42 & 0.33 & 0.72 & 0.92 & -0.74 & 0.73 & 1 \\
\hline
\end{tabular}


Table 5

Correlations between metals in S-clams, B-clams, SPM and surface sediment. All the correlations are highly significant $(p<0.001)$. S-clams, suspended clams; B-clams, bottom clams; SPM, settled particulate matter; Sed., sediment

\begin{tabular}{|c|c|c|c|c|}
\hline & S-clams & B-clams & SPM & Sed. \\
\hline \multicolumn{5}{|l|}{ Total } \\
\hline S-clams & 1 & & & \\
\hline B-clams & 0.95 & 1 & & \\
\hline SPM & 0.78 & 0.67 & 1 & \\
\hline Sed. & 0.74 & 0.65 & 0.98 & 1 \\
\hline \multicolumn{5}{|l|}{ Marano } \\
\hline S-clams & 1 & & & \\
\hline B-clams & 0.99 & 1 & & \\
\hline SPM & 0.93 & 0.89 & 1 & \\
\hline Sed. & 0.99 & 0.98 & 0.96 & 1 \\
\hline \multicolumn{5}{|l|}{ Fusina } \\
\hline S-clams & 1 & & & \\
\hline B-clams & 0.88 & 1 & & \\
\hline SPM & 0.98 & 0.81 & 1 & \\
\hline Sed. & 0.98 & 0.92 & 0.97 & 1 \\
\hline \multicolumn{5}{|c|}{ San Giuliano } \\
\hline S-clams & 1 & & & \\
\hline B-clams & 0.93 & 1 & & \\
\hline SPM & 0.98 & 0.94 & 1 & \\
\hline Sed. & 0.98 & 0.92 & 1.00 & 1 \\
\hline
\end{tabular}

As a result, at the end of the experimental period the biggest clams (Fig. 2) were found at Fusina and they referred to B-clams, whereas the smallest ones were recorded at Marano where the trophic level was low and SPM rates were ca: $200-300 \mathrm{~kg} \mathrm{~m}^{-2} \mathrm{y}^{-1}$, ca. $20-30 \%$ of the SPM rates measured at San Giuliano and Fusina (Sfriso et al., 2005a,b).

Except for $\mathrm{Hg}$ and in part for As, metal concentrations were higher in the sediments and SPM from the Venice stations, but in some cases clam bioaccumulation was not related to the

Table 6

Pearson's coefficients between metals in S-clams, B-calms, SPM and surface sediments. $p<0.05=\mathrm{X} ; p<0.001=\mathrm{XX}$. S-clams, suspended clams; B-clams, bottom clams; SPM, settled particulate matter; Sed., sediment

\begin{tabular}{|c|c|c|c|}
\hline \multirow[t]{3}{*}{$\mathrm{Cd}$} & Total & $\begin{array}{l}\text { S-clams-B-clams } \\
\text { Sed.-SPM }\end{array}$ & $\begin{array}{l}X X \\
X X\end{array}$ \\
\hline & Fusina & Sed.-SPM & $\mathrm{X}$ \\
\hline & San Giuliano & S-clams-Sed. & $\mathrm{X}$ \\
\hline \multirow[t]{3}{*}{$\mathrm{Fe}$} & Total & Sed.-SPM & $\mathrm{XX}$ \\
\hline & Marano & S-clams-B-clams & $\mathrm{X}$ \\
\hline & San Giuliano & S-clams-SPM & $\mathrm{X}$ \\
\hline \multirow[t]{3}{*}{$\mathrm{Hg}$} & Total & S-clams-B-clams & $\mathrm{XX}$ \\
\hline & & Sed.-SPM & $\mathrm{X}$ \\
\hline & San Giuliano & S-clams-Sed. & $\mathrm{X}$ \\
\hline \multirow[t]{3}{*}{$\mathrm{Ni}$} & Total & S-clams-B-clams & $\mathrm{X}$ \\
\hline & & Sed.-SPM & $\mathrm{X}$ \\
\hline & San Giuliano & Sed.-SPM & $\mathrm{X}$ \\
\hline \multirow[t]{3}{*}{$\mathrm{Zn}$} & Total & S-clams-Sed. & $\mathrm{XX}$ \\
\hline & Marano & S-clams-Sed. & $\mathrm{X}$ \\
\hline & Fusina & S-clams-SPM & $\mathrm{X}$ \\
\hline \multirow[t]{2}{*}{ As } & Total & S-clams-B-clams & $\mathrm{X}$ \\
\hline & Fusina & S-clams-SPM & $\mathrm{X}$ \\
\hline \multirow[t]{2}{*}{$\mathrm{Cu}$} & Total & Sed.-SPM & $\mathrm{XX}$ \\
\hline & Fusina & Sed.-SPM & $\mathrm{X}$ \\
\hline \multirow[t]{2}{*}{$\mathrm{Pb}$} & Total & Sed.-SPM & $\mathrm{XX}$ \\
\hline & Fusina & Sed.-SPM & $\mathrm{XX}$ \\
\hline Co & Total & Sed.-SPM & $\mathrm{XX}$ \\
\hline $\mathrm{Cr}$ & Total & Sed.-SPM & $\mathrm{X}$ \\
\hline Mn & Total & Sed.-SPM & $\mathrm{X}$ \\
\hline
\end{tabular}

Table 7

Extraction of principal components. Eigen., Eigenvalue; Cumul., cumulative

\begin{tabular}{lcrr}
\hline Significant loading $>|0.7|$ & & \\
\hline & Components & \multicolumn{1}{c}{} \\
\cline { 2 - 4 } & 1 & 2 & 0.39 \\
\hline $\mathrm{As}$ & -0.47 & -0.71 & -0.61 \\
$\mathrm{Cd}$ & 0.35 & -0.64 & 0.07 \\
$\mathrm{Cr}$ & 0.61 & 0.68 & -0.03 \\
$\mathrm{Co}$ & 0.93 & -0.21 & -0.12 \\
$\mathrm{Cu}$ & 0.79 & -0.55 & -0.02 \\
$\mathrm{~Pb}$ & 0.97 & -0.17 & 0.15 \\
$\mathrm{Mn}$ & 0.77 & 0.46 & 0.09 \\
$\mathrm{Ni}$ & 0.72 & 0.59 & 0.09 \\
$\mathrm{~V}$ & 0.98 & 0.01 & -0.10 \\
$\mathrm{Zn}$ & 0.80 & -0.54 & 0.08 \\
Fe & 0.98 & 0.08 & 0.74 \\
$\mathrm{Hg}$ & 0.31 & -0.56 & 9.6 \\
Eigen. variance \% & 57.6 & 24.3 & 91.5 \\
$\mathrm{Cumul}$ variance \% & 57.6 & 82.0 &
\end{tabular}

concentrations of these sedimentary matrices. The concentration of metals in the sediments at Fusina and San Giuliano was relatively high as we can see by a comparison with the background concentrations estimated for some metals present in the deepest strata of radio-dated cores (pre-industrial values at ca. $0.8-1 \mathrm{~m}$ depth) by Pavoni et al. (1992) and Bernardello et al. (2006). These values in $\mu \mathrm{g} \mathrm{g}^{-1} \mathrm{dwt}$ are: As: 10, Hg: 0.1, Cd: 1.0, Cr 20, Cu: 20, Pb: 25, Co: 15 , Ni: 20, Zn: 70 , and Fe: 20,000. The contamination was particularly evident for $\mathrm{Hg}$ (up to $2.3 \mu \mathrm{g} \mathrm{g}^{-1} \mathrm{dwt}$ ) in the sediment and SPM of all the three stations and for $\mathrm{Ni}$ (up to $83 \mu \mathrm{g} \mathrm{g}^{-1} \mathrm{dwt}$ ) and $\mathrm{Zn}$ (up to $330 \mu \mathrm{g} \mathrm{g}^{-1} \mathrm{dwt}$ ) at Fusina and San Giuliano. Cadmium (up to $1.9 \mu \mathrm{g} \mathrm{g}^{-1} \mathrm{dwt}$ ), Cr (up to $60 \mu \mathrm{g} \mathrm{g}^{-1} \mathrm{dwt}$ ), As (up to $22.9 \mu \mathrm{g} \mathrm{g}^{-1} \mathrm{dwt}$ ), $\mathrm{Cu}$ (up to $51.5 \mu \mathrm{g} \mathrm{g}^{-1} \mathrm{dwt}$ ), $\mathrm{Pb}$ (up to $57.1 \mu \mathrm{g} \mathrm{g}^{-1} \mathrm{dwt}$ ) and Fe (up to $23,446 \mu \mathrm{g} \mathrm{g}^{-1} \mathrm{dwt}$ ) were particularly high at San Giuliano. However, there is no relationship between metals in sediments, SPM and clam soft tissues (Table 6). In fact, As and $\mathrm{Hg}$ bio-accumulated by clams at Marano were higher than in Venice stations, although in the sedimentary matrices they were similar or even lower (Table 3 ). However, As is toxic only if present in its inorganic forms (as arsenate and arsenite), which are usually lower than $10 \%$ of the total, so there should be no risk for human health (Argese et al., in preparation). Mercury concentrations show very high concentrations (4.1$6.6 \mu \mathrm{g} \mathrm{g}^{-1}$ ) in the surface sediments of other areas of Marano and they get even higher in Grado lagoon (up to $14-15 \mu \mathrm{g} \mathrm{g}^{-1}$ ), the north-eastern part of the Marano-Grado basin. At Marano Hg contamination is caused by the Torviscosa industrial complex, which produces cloro-soda and cellulose from cane (Arundo donax) and is conveyed in the lagoon by the river Aussa-Corno mainly in organic form (up to 98\%, Piani et al., 2005). This suggests that $\mathrm{Hg}$ could be involved in biogeochemical transformations and bioaccumulation. At Grado the contamination is mainly of natural origin. Mercury is produced by Idrja mines in Slovenia and conveyed in the lagoon by the River Isonzo. However, in the station selected at Marano near the Lignano inlet, sediment contamination by $\mathrm{Hg}$ is relatively low and no significant correlations between clams, sediment and SPM were found (Table 6). Studies on the speciation of this element are in progress in order to understand what the amount of the toxic organic fraction in clam tissues is.

Cadmium was bio-accumulated mostly at Fusina, but also at Marano, where it reached values 3-4 times higher in clam tissues than in the surface sediments and SPM (Table 3). In contrast, at San Giuliano clams showed concentrations a little lower than in the sediments and SPM, even though in that station the highest $\mathrm{Cd}$ concentration recorded in the environment was found (Table 6). A significant correlation between the concentrations recorded in S-clams and in the surface sediment was also recorded. 
Lead, another toxic element considered by the law, showed a significant bioaccumulation only between the seeding and the $1 \mathrm{st}$ growth period whereas in the other periods contrasting results were recorded (Table 3). No significant correlation between clams and the sedimentary matrices was found.

The highest $\mathrm{Hg}$ clam concentrations recorded at Marano (0.28$0.30 \mu \mathrm{g} \mathrm{g}^{-1}$ fwt $)$ and the $\mathrm{Pb}$ and $\mathrm{Cd}$ peak concentrations found in the clams at Fusina, which reached 0.38 and $0.33 \mu \mathrm{g} \mathrm{g}^{-1} \mathrm{fwt}$, at the maximum, were lower than the reference values for fresh weight molluscs set out in the CEE Reg. No. 1881/2006 (Hg: $0.5 \mu \mathrm{g} \mathrm{g}^{-1} \mathrm{fwt}$, Cd: $1 \mu \mathrm{g} \mathrm{g}^{-1}$ fwt and Pb: $1.5 \mu \mathrm{g} \mathrm{g}^{-1}$ fwt).

Literature on metals and mollusks from the Venice lagoon contains a number of papers dealing with bioaccumulation in Mytilus galloprovincialis, Crassostrea gigas and Cerastoderma glaucum (Sfriso et al., 1995; Da Ros et al., 2000; Irato et al., 2003; Pojana et al., 2003; Marin et al., 2006) but only few of them considering also Tapes philippinarum. Marin et al. (2006) studied the $\mathrm{Cd}, \mathrm{Cu}, \mathrm{Ni}$, $\mathrm{Pb}, \mathrm{Zn}$ content in the surface sediments and in the digestive gland tissues of some clams, among which T. philippinarum from some stations close to Chioggia center and Marghera industrial district. Those authors found an evident bioaccumulation of $\mathrm{Zn}$ that in natural specimens reached ca. $150 \mu \mathrm{g} \mathrm{g}^{-1} \mathrm{dwt}$. In the clam exposed at Fusina and San Giuliano the concentrations of $\mathrm{Zn}$ were similar reaching $122 \mu \mathrm{g} \mathrm{g}^{-1} \mathrm{dwt}$. That value doubles the concentration found in the seed, but it is lower than the sediment and SPM. However, it refers to the total of the clam tissues and obviously the values for the digestive glands are expected to be higher. By analyzing the digestive glands and gills of T. philippinarum collected in the stations of Chioggia and Marghera Irato et al. (2003) found a bioaccumulation also for $\mathrm{Cr}\left(5.3-10.4 \mu \mathrm{g} \mathrm{g}^{-1} \mathrm{dwt}\right)$ and $\mathrm{Co}$ (14.3$54 \mu \mathrm{g} \mathrm{g}^{-1} \mathrm{dwt}$ ) which were $2-3$ and up to 22 times higher than in the total tissues of the clams exposed at Fusina and San Giuliano. Pojana et al. (2003) reported an Hg concentration in natural samples of $T$. philippinarum ranging from 0.02 to $0.11 \mu \mathrm{g} \mathrm{g}^{-1}$ fwt. Those values were similar to the concentrations found in the clams exposed at Fusina and San Giuliano where $\mathrm{Hg}$ ranged between 0.03 and $0.07 \mu \mathrm{g} \mathrm{g}^{-1}$ fwt and were lower than the $\mathrm{Hg}$ values found in the clams exposed at Marano ( 0.22 and $0.30 \mu \mathrm{g} \mathrm{g}^{-1}$ fwt).

By comparison with the literature of other sites the concentrations of some metals reported by Liang et al. (2004) for the soft tissues of Tapes philippinarum from the Chinese Bohai Sea in the Yellow Sea, Co, $\mathrm{Pb}, \mathrm{Cu}, \mathrm{Zn}$ showed values that are very similar to the ones reported in this study. Conversely, Ni and Cd concentrations in Bohai Sea were a bit higher than in Venice and Marano stations. Liang et al. (2004) found that $\mathrm{Ni}$ was the metal, which showed the highest bioaccumulation (up to $2.97 \mu \mathrm{g} \mathrm{g}^{-1} \mathrm{fwt}$ ). That value was ca. twice as high as the maximum concentration found in B-clams at Marano (1.24 $\mathrm{\mu g} \mathrm{g}^{-1} \mathrm{fwt}$ ). Comparable results were found by Usero et al. (1997) for clams from the southern Spanish Atlantic Coast, which examined clam concentrations of $\mathrm{As}, \mathrm{Hg}, \mathrm{Cd}, \mathrm{Pb}, \mathrm{Cu}, \mathrm{Zn}, \mathrm{Mn}, \mathrm{Ni}, \mathrm{Cr}$ and $\mathrm{Fe}$.

By considering metal bioaccumulation during clam growth we obtain different results for different contaminants with marked seasonal changes. Metals in the clams increased during the growth from seeding to adult-size with the exception of some elements that showed an inverse trend. On an average, at Marano metal concentrations between seeding and the first clam growth period increased markedly, except for $\mathrm{Zn}$. In the 2 nd and 3rd periods clam concentrations at Marano were higher than seed concentrations for all the considered elements. A clam concentration increase was recorded at Fusina except for As, $\mathrm{Cr}, \mathrm{Co}$ and $\mathrm{Hg}$ and at San Giuliano except for $\mathrm{As}, \mathrm{Cr}, \mathrm{Ni}$, and $\mathrm{Hg}$. During the 2 nd and $3 \mathrm{rd}$ periods $\mathrm{As}, \mathrm{Cr}$, $\mathrm{Ni}$ and $\mathrm{Hg}$ were similar or even lower. Usero et al. (1997) found similar contrasting results in the soft tissues of clams from the Atlantic Coast of southern Spain. Those authors found some metal concentration increase with the increasing of clam-size for $\mathrm{As}, \mathrm{Hg}$, $\mathrm{Pb}, \mathrm{Ni}, \mathrm{Cd}$ and an inverse trend for $\mathrm{Cu}, \mathrm{Zn}$, and $\mathrm{Fe}$.
Finally, both S-clam and B-clam concentrations showed a high correlation with the concentrations found in SPM rather than surface sediments (Table 5). The results reported in Table 6 provide evidence that, in general, S-clam concentrations are more strictly correlated to SPM and sediment concentrations than B-clams, but there are differences due to the station location.

\section{Acknowledgements}

The authors thank the Italian Ministry of Agricultural and Forestry Policies (MIPAF) that funded the present project in the framework of the "VI Triennial Plan for Fish and Aquaculture in Marine and Brackish Waters". The authors are grateful to Dr. Aurelio Zentilin for the technical assistance and to Dr. Orietta Zucchetta for the English editing. Particular thanks are also addressed to the anonymous referees for their criticisms and suggestions, which allowed preparation of the final version of this paper.

\section{References}

Argese, E., Bettiol, C., De Pol, R., Rigo, C.F., Gobbo, L., Sfriso, A. Arsenic speciation in Tapes philippinarum growing in Venice and Marano Lagoon, in preparation

Bellucci, L.G., Frignani, M., Raccanelli, S., Carraro, C., 2000. Polychlorinated dibenzo$p$-dioxins and dibenzofurans in surficial sediments of the Venice lagoon (Italy). Marine Pollution Bulletin 40, 65-76.

Bellucci, L.G., Frignani, M., Paolucci, D., Ravelli, M., 2002. Distribution of metals in sediments of the Venice lagoon: the role of the industrial area. Science of the Total Environment 295, 35-49.

Bernardello, M., Secco, T., Pellizzato, F., Chinellato, M., Sfriso, A., Pavoni, B., 2006. The changing state of contamination in the lagoon of Venice. Part 2: heavy metals. Chemosphere 64, 1334-1345.

Critto, A., Marcomini, A., 2001. Rischio ecologico e inquinamento chimico lagunare. Cafoscarina, Venezia, $162 \mathrm{pp}$

Da Ros, L., Nasci, C., Marigomez, I., Soto, M., 2000. Biomarkers and trace metal in the digestive gland of indigenous and transplanted mussels, Mytilus galloprovincialis, in Venice Lagoon, Italy. Marine Environmental Research 50, 417-423.

Donazzolo, R., Orio, A.A., Pavoni, B., Perin, G., 1984. Heavy metals in sediments of the Venice lagoon. Oceanologica Acta 7, 25-32.

Facca, C., Sfriso, A., Socal, G., 2002a. Temporal and spatial distribution of diatoms in the surface sediment of the central part of the Venice lagoon. Botanica Marina $45,170-183$.

Facca, C., Sfriso, A., Socal, G., 2002b. Changes in abundance and composition of phytoplankton and microphytobenthos due to increased sediment fluxes in the Venice lagoon, Italy. Estuarine, Coastal and Shelf Science 54, 773-792.

Guerzoni, S., Raccanelli, S., 2003. The Wounded Lagoon. Observations on the Dioxin and the Other Organic Persistent Pollutant (POP) in Venice. Cafoscarina, Venezia, 95 p. (in Italian).

Irato, P., Santovito, G., Cassini, A., Piccinini, E., Albergoni, V., 2003. Metal accumulation and binding protein induction in Mytilus galloprovincialis, Scafarca inaequivalvis and Tapes philippinarum from the Lagoon of Venice. Archives of Environmental Contamination and Toxicology 44, 476-484.

Liang, L.N., He, B., Jiang, G.B., Chen, D.Y., Yao, Z.W., 2004. Evaluation of mollusks as biomonitors to investigate heavy metal contamination along the Chinese Bohai Sea. Science of the Total Environment 324, 105-113.

Ministry of Environment, 1993. Environmentally safe criteria to excavate, transport and reuse sediments from the Venice canals. $23 \mathrm{pp}$ (in Italian).

Marcomini, A., Zanette, M., D’Andrea, F., Della Sala, S., 1997. Dioxin, Environment and Health. Arsenale Editrice, Venezia, 102 pp. (in Italian)

Marin, M.G., Boscolo, R., Cella, A., Degetto, S., Da Ros, L., 2006. Field validation of autometallographical black silver deposit (BSD) extent in three bivalve species from the Lagoon of Venice, Italy (Mytilus galloprovincialis, Tapes philippinarum, Scafarca inaequivalvis) for metal bioavailability assessment. Science of the Total Environment 371, 156-167.

Matassi, G., Rossin, P., Giacomich, P., 2007. Marano and Grado Lagoons. Synoptic framework and problems related to the implementation of WED 2000/60. Guidelines and Cases Studies for the Management of Natura 2000 Sites in Transitional Environments. In: Proceedings of the Grado Workshop 7-8 June 2006. Edizioni Università di Trieste, Trieste, pp. 296-305.

Orel, G., Boatto, V., Sfriso, A., Pellizzato, M., 2000. Management Plan for the Alieutic Resources of the Lagoons in the Province of Venice. Provincia di Venezia, Sannioprint, Benevento, 102 pp.

Pavoni, B., Marcomini, A., Sfriso, A., Donazzolo, R., Orio, A.A., 1992. Changes in an estuarine ecosystem. The lagoon of Venice as a case study. In: Dunnette, D.A., O'Brien, R.J. (Eds.), Science of Global Change. American Chemical Society, Washington, D.C., U.S.A., pp. 287-305

Piani, R., Covelli, S., Biester, H., 2005. Mercury contamination in Marano Lagoon (Northern Adriatic sea, Italy): source identification by analyses of $\mathrm{Hg}$ phases. Applied Geochemistry 20, 1546-1559.

Pojana, G., Critto, A., Micheletti, C., Carlon, C., Busetti, F., Marcomini, A., 2003. Analytical and environmental chemistry in the framework of risk assessment and 
management: the lagoon of Venice as a case study. Environmental Analysis 57, 542-549.

Pranovi, F., Giovanardi, O., 1994. The impact of hydraulic dredging for short-necked clams, Tapes spp., on an infaunal community in the lagoon of Venice. Scientia Marina 58, 345-353.

Secco, T., Pellizzato, F., Sfriso, A., Pavoni, B., 2005. The changing state of contamination in the lagoon of Venice. Part 1. Organic pollutants. Chemosphere 58, 279-290.

Sfriso, A., Marcomini, A., Zanette, M., 1995. Heavy metals in sediments, settled particulate matter and phytozoobenthos of the Venice lagoon. Marine Pollution Bulletin 30, 116-124.

Sfriso, A., Facca, C., Ceoldo, S., Silvestri, S., Ghetti, P.F., 2003. Role of macroalgal biomass and clam fishing on spatial and temporal changes in $\mathrm{N}$ and $\mathrm{P}$ sedimentary pools in the central part of the Venice lagoon. Oceanologica Acta 26, 3-13.

Sfriso, A., Facca, C., Marcomini, A., 2005a. Sedimentation fluxes and erosion processes in the lagoon of Venice. Environment International 31, 983-992.
Sfriso, A., Facca, C., Ceoldo, S., Pessa, G., 2005b. Sedimentation rates, erosive processes, grain-size and sediment density changes in the lagoon of Venice. In: Campostrini, P. (Ed.), Scientific Research and Safeguarding of Venice. CORILA Research Program 2003 Results. IVSLA, La Garangola, pp. 2003-2013.

Sfriso, A., Facca, C., Ceoldo, S., Marcomini, A., 2005c. Recording the occurrence of trophic level changes in the lagoon of Venice over the '90s. Environment International 31, 993-1001.

Sfriso, A., Favaretto, M., Ceoldo, S., Facca, C., Marcomini, A., 2005d. Organic carbon changes in the surface sediments of the Venice Lagoon. Environment International 31, 1002-1010.

Socal, G., Bianchi, F., Alberighi, A., 1999. Effects of thermal pollution and nutrient discharges on a spring phytoplankton bloom in the industrial area of the lagoon of Venice. Vie et Milieu 49, 19-31.

Usero, J., Gonzales-Regalado, E., Gracia, I., 1997. Trace metals in the bivalve molluscs Ruditapes decussatus and Ruditapes philippinarum from the Atlantic coast of southern Spain. Environment International 23, 291-298. 